\title{
An Asymptotic Analysis of Solutions of a Second Order Nonlinear Differential Equation
}

\author{
By \\ Takaŝi Kusano, Jelena V. ManoJlović and Vojislav Marić \\ (Hiroshima University, Japan, University of Niš and Serbian Academy of \\ Science and Arts, Serbia)
}

\begin{abstract}
The existence and the precise asymptotic behavior for $t \rightarrow \infty$ of all increasing solutions of a class of second order nonlinear equations is proved. For that the use of Karamata class of regularly varying functions is essential.

Key Words and Phrases. Equations of Thomas-Fermi type, Regularly varying functions, Increasing and regularly varying solutions, Asymptotic behavior.

2010 Mathematics Subject Classification Numbers. 34C11, 34A34, 34E05, $26 \mathrm{~A} 12$.
\end{abstract}

\section{Introduction}

We study here the existence and the precise asymptotic behavior when $t \rightarrow \infty$ of positive solutions $x=x(t)$ defined on a positive half-axis $a>0$ (called also proper solutions) of the equation

$$
x^{\prime \prime}=g(t) \phi(x),
$$

in the framework of regular variation in the sense of Karamata [6].

The books $[2,33]$ are the main references for the theory of regular variation but to help the reader we shall present here some basics.

Definition 1.1. A measurable function $f:[a, \infty) \rightarrow(0, \infty), a>0$, is said to be regularly varying at infinity of index $\rho \in \boldsymbol{R}$ if

$$
\lim _{t \rightarrow \infty} \frac{f(\lambda t)}{f(t)}=\lambda^{\rho} \quad \text { for all } \lambda>0 .
$$

With $\operatorname{RV}(\rho)$ we denote, the set of regularly varying functions of index $\rho$ at infinity. If in particular, $\rho=0$, the function $f$ is called slowly varying at infinity. With SV we denote, the set of these. Saying only regularly or slowly varying function, we mean regularity at infinity.

It follows from Definition 1.1 that any function $f(t) \in \mathrm{RV}(\rho)$ is written as $f(t)=t^{\rho} \ell(t)$, with $\ell(t) \in \mathrm{SV}$. If in particular the function $\ell(t) \rightarrow k>0$ as $t \rightarrow \infty$, it is called a trivial slowly varying one denoted by $\ell(t) \in t r-\mathrm{SV}$, in which 
case the function $f(t) \in \mathrm{RV}(\rho)$ is called a trivial regularly varying of index $\rho$, denoted by $f(t) \in t r-\operatorname{RV}(\rho)$. Otherwise $\ell(t)$ is called a nontrivial slowly varying function and $f(t)$ is called a nontrivial $\mathrm{RV}(\rho)$ function.

A more general class is introduced by

Definition 1.2. A measurable function $f:[a, \infty) \rightarrow(0, \infty), a>0$ is said to be regularly bounded at infinity (or R-O varying) if for $t \geq T$ and for each $1 \leq \lambda \leq \lambda_{0}$

$$
m \leq \frac{f(\lambda t)}{f(t)} \leq M
$$

where $\lambda_{0}, m, M$ are any constants such that $1<\lambda_{0}<\infty, 0<m<1$, $1<M<\infty$.

E.g. any measurable positive function which is bounded away from both 0 and $\infty$ on $[a, \infty)$ is such and it is obvious that any $\mathrm{RV}$ function is regularly bounded.

A related class is introduced by

Definition 1.3. A positive function $f(t)$ is said to be almost increasing (decreasing) if there exist a constant $A>1$ such that $t_{2}<t_{1}$ implies $f\left(t_{2}\right) \leq A f\left(t_{1}\right)\left(f\left(t_{1}\right) \leq A f\left(t_{2}\right)\right)$.

Such study of asymptotics of solutions of differential equations via regular variation was initiated in the seminal paper of V. G. Avakumović [1] and about 30 years later extended and developed in [24]. Then, after the monograph [23] appeared, numerous papers in that spirit have been published, dealing also with some more general differential equation of the second order, the ones of higher orders and some systems, functional differential equations, difference and dynamic ones and also some partial differential ones (see e.g. [7]-[22], [24]-[32], [34]-[35]). In [5] V. M. Evtukhov and A. M. Samoilenko by a different approach studied the $n$-th order equation $x^{(n)}=\alpha_{0} g(t) \phi(x)$ for both $\alpha_{0}= \pm 1$

In what follows the symbol $\sim$ denotes the asymptotic equivalence

$$
f(t) \sim g(t), \quad t \rightarrow \infty \quad \Leftrightarrow \quad \lim _{t \rightarrow \infty} \frac{f(t)}{g(t)}=1 .
$$

Also, $f(t) \asymp g(t)$ means that there exist constants $0<m<M$ such that

$$
m g(t) \leq f(t) \leq M g(t), \quad t \geq T .
$$

The following results on regularly varying functions (and of some generalizations of these) will be used throughout the paper. 
Proposition 1.1 (Karamata's integration theorem). Let $L(t) \in \mathrm{SV}$. Then, (i) if $\alpha>-1$,

$$
\int_{a}^{t} s^{\alpha} L(s) d s \sim \frac{1}{\alpha+1} t^{\alpha+1} L(t), \quad t \rightarrow \infty ;
$$

(ii) if $\alpha<-1$,

$$
\int_{t}^{\infty} s^{\alpha} L(s) d s \sim-\frac{1}{\alpha+1} t^{\alpha+1} L(t), \quad t \rightarrow \infty
$$

(iii) if $\alpha=-1$,

$$
\begin{array}{ll}
m_{1}(t)= & \int_{a}^{t} \frac{L(s)}{s} d s \in \mathrm{SV}, \quad m_{2}(t)=\int_{t}^{\infty} \frac{L(s)}{s} d s \in \mathrm{SV} \\
\text { and } \quad \lim _{t \rightarrow \infty} \frac{L(t)}{m_{i}(t)}=0, \quad i=1,2 .
\end{array}
$$

We shall also use the following results (see [23, Appendix]):

Proposition 1.2. Let $q_{1}(t) \in \mathrm{RV}\left(\sigma_{1}\right), q_{2}(t) \in \mathrm{RV}\left(\sigma_{1}\right)$.

$$
\begin{aligned}
& q_{1}(t) q_{2}(t) \in \operatorname{RV}\left(\sigma_{1}+\sigma_{2}\right),\left(q_{1}(t)\right)^{\alpha} \in \operatorname{RV}\left(\alpha \sigma_{1}\right) \quad \text { for any } \alpha \in R \\
& q_{1}\left(q_{2}(t)\right) \in \operatorname{RV}\left(\sigma_{1} \sigma_{2}\right) \quad \text { if } q_{2}(t) \rightarrow \infty \text {, as } t \rightarrow \infty .
\end{aligned}
$$

Proposition 1.3. If $f(t) \sim t^{\alpha} \ell(t)$ as $t \rightarrow \infty$ with $\ell(t) \in \mathrm{SV}$, then $f(t)$ is a regularly varying function of index $\alpha$, i.e. $f(t)=t^{\alpha} \ell^{*}(t), \ell^{*}(t) \in \mathrm{SV}$, where in general $\ell^{*}(t) \neq \ell(t)$, but $\ell^{*}(t) \sim \ell(t)$ as $t \rightarrow \infty$.

Proposition 1.4. Let $f(t)$ be a positive, continuously differentiable for $t>0$ and such that

$$
\lim _{t \rightarrow \infty} \frac{t f^{\prime}(t)}{f(t)}=0
$$

Then, $f(t)$ is slowly varying.

Proposition 1.5. (i) Regularly varying function $f(t)$ of index $\rho \neq 0$ is almost monotone.

(ii) A positive continuous function $f(t)$ defined on $[a, \infty)$ is regularly bounded at $\infty$ if and only if there exist real numbers $p, q, p>q$ such that $t^{p} f(t)$ is almost increasing and $t^{q} f(t)$ is almost decreasing for sufficiently large $t$.

Proposition 1.6. For the function $f(t) \in \mathrm{RV}(\alpha), \alpha>0$, there exists $g(t) \in$ $\mathrm{RV}(1 / \alpha)$ such that

$$
f(g(t)) \sim g(f(t)) \sim t \quad \text { as } t \rightarrow \infty .
$$


Here, $g$ is an asymptotic inverse of $f$ (and it is determined uniquely to within asymptotic equivalence).

Proposition 1.7 [3, Theorem 3]. Let $x, y$ be arbitrary positive functions on $[a, \infty)$ such that $x(t) \rightarrow \infty, y(t) \rightarrow \infty$ and $x(t) \sim y(t)$ as $t \rightarrow \infty$. Then for any regularly varying function $F$ it holds

$$
F(x(t)) \sim F(y(t)), \quad t \rightarrow \infty .
$$

Throughout the paper it is assumed that the functions $g(t)$ and $\phi(x)$ are continuous and positive for $t \in(a, \infty)$ for some $a>0$, and $x \in(0, \infty)$, respectively. Further, we suppose that

$$
\begin{array}{lll}
g(t) \in \operatorname{RV}(\sigma), & \sigma \in R, & g(t)=t^{\sigma} \ell(t), \quad \ell \in \mathrm{SV} ; \\
\phi(x) \in \operatorname{RV}(\gamma), \quad \gamma>1, & \phi(x)=x^{\gamma} L(x), \quad L \in \mathrm{SV} .
\end{array}
$$

in which case the considered equation $(\mathrm{A})$ can be written in the form

$$
x^{\prime \prime}(t)=t^{\sigma} \ell(t) x(t)^{\gamma} L(x(t))
$$

where $\ell(t) \in \mathrm{SV}, L(x) \in \mathrm{SV}$. However, the results in Section 3 are proved for the more general class of regularly bounded functions $g$ and $\phi$.

Equation (1.3) is called sublinear or superlinear according as $\gamma<1$ or $\gamma>1$. If $\gamma=1, L(x) \neq$ const. it may be called quasi- or pseudo-linear.

First observe that all (positive) proper solutions $x(t)$ are convex. It follows that $x^{\prime}(t)$ is increasing and so on $[T, \infty)$ either $x^{\prime}(t)<0$ or $x^{\prime}(t)>0$. The former case leads to decreasing solutions and the later to the increasing ones.

In the later case, since $x^{\prime}(t)$ is positive and increasing, $x^{\prime}(t) \geq x^{\prime}\left(t_{1}\right)$ for $t \geq t_{1}$ and integration over $\left(t_{1}, t\right)$ gives $x(t) \geq x\left(t_{1}\right)+x^{\prime}\left(t_{1}\right)\left(t-t_{1}\right)$, implying that $x(t) \rightarrow \infty, t \rightarrow \infty$ increases and so all possible positive, increasing solutions belong to either one of following two classes:

$$
\begin{array}{ll}
\text { (i) } \lim _{t \rightarrow \infty} x(t)=\infty, & \lim _{t \rightarrow \infty} x^{\prime}(t)=\lim _{t \rightarrow \infty} \frac{x(t)}{t}=\text { const. }>0 \\
\text { (ii) } \lim _{t \rightarrow \infty} x(t)=\infty, \quad \lim _{t \rightarrow \infty} x^{\prime}(t)=\lim _{t \rightarrow \infty} \frac{x(t)}{t}=\infty .
\end{array}
$$

For the sublinear equation the existence and the precise asymptotic behavior for $t \rightarrow \infty$ of both mentioned type of solutions are obtained in [22] and [18]. The quasi (pseudo) linear case is treated in [4] and [31]. For the superlinear equation, the existence of decreasing solution is proved in [36] and the precise asymptotic behavior is obtained in [23]. The remaining case, that is similar results for increasing solution of the superlinear equation 
is likely to be most demanding one. So far only the special case $\phi(x)=x^{\gamma}$ has been resolved: the existence in [28] and the asymptotic behavior in [19].

In the present paper we prove both of these results for the equation (A) in the form (1.3).

Throughout the text " $t \geq T$ " means that $t$ is sufficiently large, so that $T$ need not to be the same at each occurrence. Also, all minimizing constants will be denoted by the same letter $m$ and all majorizing ones by $M$.

The following result of more general nature will be also needed (for the proof see [19]).

Lemma 1.1. Let $g(t)$ be a positive, twice continuously differentiable convex function on $(0, \infty)$ tending to $\infty$ as $t \rightarrow \infty$. If

$$
\frac{g\left(t_{0}\right)}{g\left(k t_{0}\right)}<1-m
$$

for some $t_{0}>0, k>1$ and $m \in(0,1)$, then

$$
\frac{g(t)}{g(k t)}<\frac{1}{1+m} \quad \text { for all } t \in\left[t_{0}, k t_{0}\right] .
$$

Remark 1.1. If in Lemma 1.1 one takes $g(t)=t y(t), y(t)$ increasing to $\infty$ and satisfying $y\left(t_{i}\right) / y\left(k t_{i}\right)<\alpha<1, i=1,2, \ldots$, then (1.5) holds with $m=$ $1-\alpha / k$. Hence, an application of the Lemma 1.1 to $g(t)$ gives $y(t) / y(k t)<r$ for all $t \in\left[t_{i}, k t_{i}\right]$ where $r=k^{2} /(2 k-\alpha)$, implying $r<1$ for $1<k<1+\sqrt{1-\alpha}$.

\section{The existence}

The case (1.4)(i) is completely resolved by the following

Theorem 2.1. Assume that, $g:[a, \infty) \rightarrow(0, \infty)$ is a continuous function; $\phi:[0, \infty) \rightarrow(0, \infty)$ is a continuous regularly varying function of index $\gamma>1$. Then equation (A) possesses positive increasing solutions of the class (1.4)(i) if and only if

$$
\int_{a}^{\infty} g(t) \phi(t) d t<\infty .
$$

Proof. (The "only if" part) Suppose that (A) has a solution $x(t)$ of the class (1.4)(i) i.e. such that $x^{\prime}(t) \rightarrow c>0$ as $t \rightarrow \infty$. Integrating (A) on $[t, \infty)$ gives

$$
x^{\prime}(t)=c-\int_{t}^{\infty} g(s) \phi(x(s)) d s,
$$


which implies the convergence of the above integral. Since $\phi \in \operatorname{RV}(\gamma)$ and $x(t) \sim c t, t \rightarrow \infty$, Proposition 1.7 and (1.1) imply

$$
\phi(x(t)) \sim \phi(c t) \sim c^{\gamma} \phi(t), \quad t \rightarrow \infty,
$$

so that $T$ can be chosen so large that $\phi(x(t)) \geq \frac{1}{2} c^{\gamma} \phi(t), t \geq T$. Using this fact, we obtain

$$
\infty>\int_{T}^{\infty} g(s) \phi(x(s)) d s \geq \frac{1}{2} c^{\gamma} \int_{T}^{\infty} g(s) \phi(s) d s,
$$

which confirms the truth of (2.1).

(The "if" part) Assume that (2.1) is satisfied. Applying Proposition 1.5 to the function $\phi(x) \in \operatorname{RV}(\gamma)$ with $\gamma>0$, we see that there exists a constant $A>1$ such that

$$
\phi(x) \leq A \phi(y) \quad \text { for each } a>y \geq x>0 .
$$

Since $(1.1)$ holds uniformly on each compact $\lambda$-set on $(0, \infty)$ (see $[2$, Theorem 1.2.1]), there exists $T>a$ such that

$$
\phi(c t) \leq c^{\gamma} \phi(t), \quad \text { for } t \geq T \text { and all } c>0 .
$$

In view of (2.1) we may choose $T>a$ such that

$$
\int_{T}^{\infty} g(s) \phi(s) d s \leq \frac{c^{1-\gamma}}{2 A} .
$$

Consider the integral operator

$$
\mathscr{F} x(t)=c t-\int_{T}^{t} \int_{s}^{\infty} g(r) \phi(x(r)) d r d s, \quad t \geq T,
$$

and let it act on the set

$$
\chi=\left\{x \in C[T, \infty): \frac{1}{2} c t \leq x(t) \leq c t, t \geq T\right\} .
$$

If $x \in \chi$, then using (2.2), (2.3) and (2.4), we see that

$$
\begin{aligned}
\int_{T}^{t} \int_{s}^{\infty} g(r) \phi(x(r)) d r d s & \leq t \int_{T}^{\infty} g(s) \phi(x(s)) d r d s \\
& \leq A t \int_{T}^{\infty} g(s) \phi(c s) d s \leq A c^{\gamma} t \int_{T}^{\infty} g(s) \phi(s) d s \leq \frac{1}{2} c t
\end{aligned}
$$

and hence that

$$
\frac{1}{2} c t \leq \mathscr{F} x(t) \leq c t, \quad t \geq T .
$$


Thus, $\mathscr{F}$ is a self-map on $\chi$. It can be verified without difficulty that $\mathscr{F}$ is continuous and $\mathscr{F}(\mathscr{X})$ is relatively compact subset in the topology of the locally convex space $C[T, \infty)$. Consequently, the Schauder-Tychonoff fixed point theorem ensures the existence of a fixed point $x \in \chi$ of $\mathscr{F}$, which clearly gives birth to a solution of equation (A) satisfying $x(t) / t \rightarrow c$ as $t \rightarrow \infty$. This completes the proof.

Thus, the class (1.4)(i) is not empty. To treat the existence of solutions of the class (1.4)(ii) we need the following result due to Wong [36, Theorem 2.5] determining whether equation

$$
x^{\prime \prime}=x F(x, t)
$$

has positive proper solutions which are asymptotically equivalent to $v$ for a given positive convex increasing function $v \in C^{2}[a, \infty)$, where

1. $F(x, t)$ is continuous in $x$ for $0 \leq x<\infty$ and in $t$ for $t \geq a$;

2. $F(x, t)>0$ for $x>0$ and $t \geq a$;

3. $F(x, t)<F(y, t)$ for each $t \geq a$ and $0<x<y<\infty$.

Proposition 2.1 (Wong [36]). Let $p(t)$ be a positive continuous function in $\left[t_{0}, \infty\right)$ and $u$ and $v$ be two linearly independent positive solutions of the linear differential equation $y^{\prime \prime}-p(t) y=0$. If, moreover, $[F(\mu v, t)-p(t)]$ is either negative for all $\mu>0$ or positive for some $\mu>0$, then a necessary and sufficient condition for equation (2.5) to have positive, convex proper solutions $x$ of the form $x(t) \sim c v(t), c>0, t \rightarrow \infty$, is that there is some $\beta>0$ such that

$$
\int_{a}^{\infty} u(t) v(t)|F(\beta v, t)-p(t)| d t<\infty .
$$

We prove

Theorem 2.2. Let $g(t) \in \mathrm{RV}(\sigma), \sigma \in \boldsymbol{R}, \phi(x) \in \mathrm{RV}(\gamma), \gamma>1$, i.e.

$$
g(t)=t^{\sigma} \ell(t), \quad \phi(x)=x^{\gamma} L(x), \quad \ell, L \in \mathrm{SV} .
$$

If

$$
\gamma+\sigma+1<0
$$

there exists slowly varying $\ell(t)$ such that there exists a solution $x(t)$ of equation (A) of the class (1.4)(ii) i.e. such that $x(t) / t \rightarrow \infty$ as $t \rightarrow \infty$.

Proof. It suffices to show that the function

$$
F(x, t):=g(t) x^{-1} \phi(x)=t^{\sigma} \ell(t) x^{\gamma-1} L(x)
$$

besides 1, 2, 3, which requires of $x^{-1} \phi(x)$ to be increasing, satisfies the conditions of Proposition 2.1. 
To that end first observe that the functions

$$
v(t)=\int_{T}^{t} \int_{T}^{s} \rho(\rho-1) r^{\rho-2} \ln r d r d s
$$

with $\rho>1$ and

$$
u(t)=v(t) \int_{t}^{\infty} v(s)^{-2} d s
$$

are two linearly independent solutions of the equation $y^{\prime \prime}-p(t) y=0$ where

$$
p(t)=\frac{v^{\prime \prime}(t)}{v(t)} .
$$

By applying Proposition 1.1 to (2.8), (2.9), (2.10) one obtains for $t \rightarrow \infty$

(2.11) $\quad v(t) \sim t^{\rho} \ln t, \quad u(t) \sim \frac{t v(t)^{-1}}{2 \rho-1} \quad$ and $\quad u(t) v(t) \sim \frac{t}{2 \rho-1}$.

so that by Proposition 1.3, $v(t)=t^{\rho} L_{1}(t)$, with $L_{1}(t) \in \mathrm{SV}$ and $L_{1}(t) \sim \ln t$, $t \rightarrow \infty$.

In addition one has

$$
p(t)=\rho(\rho-1) t^{-2} \frac{\ln t}{L_{1}(t)} .
$$

Note, integral in (2.9) converges due to (2.11) and $\rho>1$.

We have to show that for some $\beta>0, G(t)=F(\beta v(t), t)-p(t)$ is positive and

$$
\int_{a}^{\infty} u(t) v(t) G(t) d t<\infty
$$

By taking $\rho=-(\sigma+2) /(\gamma-1), \beta^{\gamma-1}=\rho(\rho-1)$ implying $\rho>1$ due to (2.6), there follows

$$
G(t)=\rho(\rho-1) \frac{\ln t}{L_{1}(t)} t^{-2}(\mathscr{L}(t)-1)
$$

where

$$
\mathscr{L}(t)=L_{1}(t)^{\gamma} L\left(\beta t^{\rho} L_{1}(t)\right) \ell(t) / \ln t
$$

Since $\ell(t)$ is arbitrary, we may choose $\ell(t)$ such that $\mathscr{L}(t)>1$ and for $t \rightarrow \infty$, $\mathscr{L}(t)=1+o(1)$, where $o(1)$ is unrestricted. 
In view of $(2.11),(2.14)$ and the choice of $\ell(t)$, there follows

$$
u(t) v(t) G(t) \sim \frac{\rho(\rho-1)}{2 \rho-1} t^{-1} o(1)
$$

and (2.13) follows by an appropriate choice of $o(1)$, which means that all conditions of Proposition 2.1 are fulfilled and so there exists a solution $x(t)$ of (A) (in the form (2.5) such that for $t \rightarrow \infty x(t) \sim c t^{\rho} \ln t, c>0$ i.e. of the class (1.4)(ii), since $\rho>1$. This proves the theorem.

Remark 2.1. Observe, Theorem 2.2 is proved under very restrictive conditions. This however, is of no concern for our further considerations, since the theorem shows that the set of solutions $x(t)$ of equation (A) of the class (1.4)(ii) is not empty.

Example 2.1. Take in the proof $L(x)=\ln x$.

Now choose $\ell(t)$ such that

$$
\ell(t)=\rho^{-1}\left(\frac{1}{(\ln t)^{\gamma}}+\frac{1}{(\ln t)^{\alpha}}\right), \quad \alpha>\gamma+1 .
$$

Hence $\mathscr{L}(t) \sim 1+(\ln t)^{\gamma-\alpha}$ and one has $u(t) v(t) G(t) \sim(\rho(\rho-1) /$ $(2 \rho-1)) t^{-1}(\ln t)^{\gamma-\alpha}$ and (2.13) follows in view of (2.15).

\section{The estimates}

In this section we give some estimates (in the form of inequalities valid for $t \geq T$ ) of all existing (positive) increasing solutions $x(t)$ of equation (A). To do it, put into $(\mathrm{A})$

$$
x(t)=t y(t)
$$

to obtain

$$
\left(t^{2} y^{\prime}\right)^{\prime}=\operatorname{tg}(t) \phi(t y(t)) .
$$

Note, in the previous section it is proved that under certain conditions positive increasing solutions exist when $g(t) \in \mathrm{RV}(\sigma), \phi(x) \in \mathrm{RV}(\gamma)$, which is more general then in [19] and also must be one of the types (1.4)(i)-(1.4)(ii).

Theorem 3.1. Let $g(t)$ and $\phi(x)$ be regularly bounded at infinity and $\phi(x)$ is such that

$$
x^{-s} \phi(x) \text { almost decreases for some } s>1
$$

and

$$
x^{-r} \phi(x) \text { almost increases for some } r<s \text { with } r>1 \text {. }
$$


If

$$
\int_{a}^{\infty} u^{s} g(u) d u<\infty, \quad s>1,
$$

then for all existing (positive) increasing solution $x(t)$ of (A) there holds: Either

$$
x(t) \sim c t, \quad \text { as } t \rightarrow \infty \text { where } c>0,
$$

or, for $t \geq T$,

$$
m\left\{t^{1-s} \int_{t}^{\infty} u^{s} g(u) d u\right\}^{-1} \leq \frac{\phi(x(t))}{x(t)} \leq M\left\{t^{1-r} \int_{t}^{\infty} u^{r} g(u) d u\right\}^{-1} .
$$

Proof. If $x(t)$ is of the class (1.4)(i), formula (3.6) holds.

If $x(t)$ is of class (1.4)(ii), in view of $x(t)=t y(t)$ implying that $y(t) \rightarrow \infty$, as $t \rightarrow \infty$, one has to prove the following inequality for large $t$

$$
m\left\{t^{-s} \int_{t}^{\infty} u^{s} g(u) d u\right\}^{-1} \leq \frac{\phi(t y(t))}{y(t)} \leq M\left\{t^{-r} \int_{t}^{\infty} u^{r} g(u) d u\right\}^{-1} .
$$

To do it, we use the method which mimics the one in [19].

To begin with, observe that in view of Proposition 1.5, there exist numbers $p, q$ with $q<p$ such that for $t \geq T$
a) $t^{q} g(t)$ almost decreases
b) $t^{p} g(t)$ almost increases

We first prove the right-hand side inequality in (3.8): To that end integrate on both sides of (3.2) over $(t, k t)$ for some $k>1$ and then use (3.9)a), (3.3) and the fact that $y(t)$ increases, to minorize the right-hand side integral. This yields

$$
y^{\prime}(k t) \geq m g(k t) \phi(k t y(k t))\left(\frac{y(t)}{y(k t)}\right)^{s} .
$$

On the other hand multiply (3.2) by $t^{2} y^{\prime}(t)$, integrate over $(t, k t)$ and use again (3.9)a), (3.3) and the fact that $y(t)$ and so $t^{2} y^{\prime}(t)$ both increase, to obtain

$$
y^{\prime}(k t)^{2} \geq m t^{-1} g(k t) y(k t)^{-s} \phi(k t y(k t)) \int_{t}^{k t} y(u)^{s} y^{\prime}(u) d u,
$$

and so

$$
y^{\prime}(k t) \geq m\left\{t^{-1} g(k t) y(k t) \phi(k t y(k t))\right\}^{1 / 2}\left\{1-\left(\frac{y(t)}{y(k t)}\right)^{s+1}\right\}^{1 / 2} .
$$


From (3.10) and (3.11) we shall derive the following inequality holding for all $t \geq T$

$$
y^{\prime}(k t) \geq m g(k t) \phi(k t y(k t)) .
$$

Obviously, the behavior of the quotient $0<y(t) / y(k t)<1$ is essential in that. For, if e.g. $\lim \sup _{t \rightarrow \infty} y(t) / y(k t)=1$, or $\lim \inf _{t \rightarrow \infty} y(t) / y(k t)=0$, inequalities (3.10), or (3.11) are useless. Therefore consider the following alternative:

Take a fixed $k>1$, (to be specified later) and an arbitrary fixed $\alpha$ such that $0<\alpha<1$. There holds:

Either

$$
\frac{y(t)}{y(k t)} \geq \alpha
$$

for all $t$ belonging to some intervals $\bar{I}_{n}, n \geq 1$ which might be all ultimately neighbouring when $\bigcup_{n=1}^{\infty} \bar{I}_{n}=[T, \infty)$ for some $T \geq a$,

or

$$
\frac{y(t)}{y(k t)}<\alpha
$$

for all $t$ belonging to some intervals $\underline{I}_{n}, n \geq 1$, which again might be all ultimately neighbouring when $\bigcup_{n=1}^{\infty} \underline{I}_{n}=[T, \infty)$ for some $T \geq a$.

In general, due to the continuity of $y(t)$, one has

$$
\bigcup_{n \geq 1}\left(\underline{I}_{n} \cup \bar{I}_{n}\right)=[T, \infty) \text {. }
$$

Such an alternative gives us the possibility to replace in the subsequent considerations the quotient $y(t) / y(k t)$ by a fixed constant.

If (3.13) holds, then (3.10) gives (3.12) for all $t \in \bar{I}_{n}$.

If, on the other hand, (3.14) holds, choose a sequence $\left\{t_{n}\right\}, n \geq 1$, of arbitrary points $t_{n} \in \underline{I}_{n}$ so that (3.14) holds for $t=t_{n}$. But then, because of Lemma 1.1, and Remark 1.1, there exists $0<\alpha^{\prime}<1$ such that $y(t) / y(k t)<\alpha^{\prime}$ for all $t \in\left[t_{n}, k t_{n}\right]$. Hence, from (3.11) using the proceeding inequality, and after dividing throughout by $(k t)^{-r / 2}(y(k t) \phi(k t y(k t)))^{1 / 2}$ and integrating over $\left[t_{n}, k t_{n}\right]$ one obtains

$$
\int_{t_{n}}^{k t_{n}} \frac{y(k u)^{-(1+r) / 2} y^{\prime}(k u) d(k u)}{(k u y(k u))^{r / 2} \phi(k u y(k u))^{1 / 2}} \geq m \int_{t_{n}}^{k t_{n}} u^{(r-p-1) / 2}\left[(k u)^{p} g(k u)\right]^{1 / 2} d u .
$$

Now, use (3.4) to majorize the left-hand side integral and (3.9)b) to minorize the right-hand side one. Upon the integration on both sides, this gives

$$
y\left(k t_{n}\right)^{1 / 2} \phi\left(k t_{n} y\left(k t_{n}\right)\right)^{-1 / 2} \geq m\left(k t_{n}\right)^{1 / 2} g\left(k t_{n}\right)^{1 / 2}
$$


and so

$$
\left\{t^{-1} g\left(k t_{n}\right) y\left(k t_{n}\right) \phi\left(k t_{n} y\left(k t_{n}\right)\right\}^{1 / 2} \geq m g\left(k t_{n}\right) \phi\left(k t_{n} y\left(k t_{n}\right)\right) .\right.
$$

By combining (3.11), (3.14) and (3.16) one obtains (3.12) for $t=t_{n}$ and so for all $t \in \underline{I}_{n}$ since $t_{n}$ is arbitrary in these intervals.

Hence, due to (3.15), inequality (3.12) holds for all $t \geq T$.

To conclude the proof of the right-hand side inequality in (3.8), divide (3.12) throughout by $t^{-r}(\phi(k t y(k t))$ integrate over $(t / k, \infty)$ and use (3.4) to majorize the left-hand side integral. Upon integration, bearing in mind $r>1$, this leads, for all $t \geq T$, to

$$
\frac{y(t)}{\phi(t y(t))} \geq m t^{-r} \int_{t}^{\infty} u^{r} g(u) d u,
$$

where the integral converges due to (3.5).

Next we prove the left-hand side inequality in (3.8): By integrating on both sides of (3.2) over $(t, k t)$ for some $k>1$, using (3.9)b), and (3.4), after dividing by $t^{2}$, one gets, since $t^{2} y^{\prime}$ increases,

$$
0<k^{2} y^{\prime}(k t)-y^{\prime}(t) \leq M g(k t) \phi(k t y(k t)) .
$$

Another integration over $(t / k, t)$, due to (3.4) leads to

$$
y(k t)\left\{1-\frac{k+1}{k} \frac{y(t)}{y(k t)}\right\} \leq M \phi(k t y(k t)) t^{-r} \int_{t / k}^{t}(k u)^{r} g(k u) d u,
$$

or, by putting $u=z / k^{2}$ and since, due to (3.9)b), $(z / k)^{r+q} g(z / k) \leq A z^{r+q} g(z)$, for some $A>1$, to

$$
\begin{aligned}
\frac{y(k t)}{\phi(k t y(k t))}\left\{1-\frac{k+1}{k} \frac{y(t)}{y(k t)}\right\} & \leq M t^{-r} \int_{k t}^{k^{2} t} z^{r+s-s} g(z) d z \\
& \leq M t^{-s} \int_{k t}^{\infty} z^{s} g(z) d z
\end{aligned}
$$

holding for all $t \geq T$.

On the other hand, divide (3.17) throughout by $(k t)^{-s} \phi(k t y(k t))$ and integrate over $(t, \infty)$ to obtain

$$
\int_{t}^{\infty} \frac{\left(k^{2} y^{\prime}(k u)-y^{\prime}(u)\right) y(k u)^{-s}}{(k u y(k u))^{-s} \phi(k u y(k u))} d u \leq M \int_{t}^{\infty}(k u)^{s} g(k u) d u .
$$

Now, since $y(k u)^{-s} \leq y(u)^{-s}$ and using (3.3), the left-hand side integral is minorized by

$$
\frac{y(k t)^{s}(k t)^{s}}{\phi(k t y(k t))}\left\{\int_{t}^{\infty} \frac{k y^{\prime}(k u)}{y(k u)^{s}} d(k u)-\int_{t}^{\infty} \frac{y^{\prime}(u)}{y^{s}(u)} d u\right\}
$$


so that upon integration, inequality (3.19) yields

$$
\frac{y(k t)}{\phi(k t y(k t))}\left\{k-\left(\frac{y(t)}{y(k t)}\right)^{1-s}\right\} \leq M t^{-s} \int_{k t}^{\infty} u^{s} g(u) d u
$$

holding for all $k t \geq T$.

At this point we use the same alternative (3.13)-(3.14) as before.

If (3.14) holds, from (3.18) one obtains

$$
\frac{k t y(k t)}{\phi(k t y(k t))} \leq M t^{1-s} \int_{k t}^{\infty} u^{s} g(u) d u
$$

which is valid for all $t \in \underline{I}_{n}$ and all $k$ such that

$$
k>\frac{\alpha}{1-\alpha}, \quad(\alpha<1) .
$$

If however, (3.13) holds, then (3.21) follows from (3.20) for all $t \in \bar{I}_{n}$ and all $k$ such that

$$
k>\alpha^{1-s} \quad(\alpha<1) .
$$

Therefore, if in view of (3.22) and (3.23) we choose

$$
k=\max \left(\frac{\alpha}{\alpha-1}, \alpha^{1-s}\right)
$$

the left-hand side inequality in (3.8) holds for all $k t>T$. This completes the proof of (3.8) and, needless to say, the estimates (3.7) for $x(t)$ since $x(t)=t y(t)$.

\section{The asymptotics}

By restricting both classes of functions $g(t)$ and $\phi(x)$ to the regularly varying ones, we can prove the more precise

Theorem 4.1. Let in equation (A) $g(t) \in \mathrm{RV}(\sigma), \sigma \in \boldsymbol{R}, \phi(x) \in \mathrm{RV}(\gamma), \gamma>1$ i.e.

$$
x^{\prime \prime}(t)=t^{\sigma} \ell(t) x(t)^{\gamma} L(x(t))
$$

then for all existing (positive) increasing solutions $x(t)$ of equation (A) of the class (1.4)(ii) i.e. such that $x(t) / t \rightarrow \infty$ as $t \rightarrow \infty$, there holds:

a) The condition

$$
\sigma<-\gamma-1
$$

is a necessary and 
b)

$$
\sigma<-s-1 \quad \text { for some } s>\gamma>1
$$

is a sufficient one for $x(t)$ to be regularly varying of index $\rho>1$ with

$$
\rho=-\frac{\sigma+2}{\gamma-1} \text {. }
$$

All these solutions have the same asymptotic behavior for $t \rightarrow \infty$

$$
\frac{\phi(x(t))}{x(t)}=x^{\gamma-1}(t) L(x(t)) \sim\left(\frac{t^{2} g(t)}{\rho(\rho-1)}\right)^{-1}
$$

i.e.

$$
x(t) \sim \psi\left(\left(\frac{t^{2} g(t)}{\rho(\rho-1)}\right)^{-1}\right)
$$

where $\psi$ is an asymptotic inverse of $x^{\gamma-1} L(x)$.

Proof. a) Let $x(t)=t^{\rho} \xi(t)$ be a solution of (A) on $[T, \infty)$ of the class (1.4)(ii) with $\rho>1$ and $\xi(t) \in \mathrm{SV}$. Then, due to Proposition 1.2, $g(s) \phi(x(s)) \in$ $\operatorname{RV}(\sigma+\rho \gamma)$ (as a function of $s$ ) and $L\left(t^{\rho} \xi(t)\right) \in \mathrm{SV}$. Thus equation (1.3) becomes of the same type as equation (A) in [19] with a different slowly varying factor on the right-hand side- $\ell(t) \xi(t)^{\gamma} L\left(t^{\rho} \xi(t)\right)$ instead of $\ell(t) \xi(t)^{\gamma}$ and one may apply [19, Thm 2.2, I, the only if part] to obtain the statement including asymptotic formula (4.5).

b) Assume (4.3) and use "." to denote differentiation with respect to $x$. Put

$$
I(t)=\int_{t}^{\infty} \frac{1}{s} \int_{s}^{\infty} u g(u) d u d s
$$

the occurring integrals converge due to (4.3). Then, by Proposition 1.1 we get for $t \rightarrow \infty$

$$
\begin{aligned}
& \text { i) } I(t) \sim \frac{1}{(\sigma+2)^{2}} t^{2} g(t), \quad \text { ii) } I^{\prime}(t) \sim \frac{1}{\sigma+2} \operatorname{tg}(t), \\
& \text { iii) } I^{\prime \prime}(t)=\frac{\sigma+1}{\sigma+2}(1+o(1)) g(t)
\end{aligned}
$$

and so
i) $\frac{g(t)}{-I^{\prime}(t)} \sim-\frac{\sigma+2}{t}$,
ii) $\frac{I^{\prime}(t)}{I(t)} \sim \frac{\sigma+2}{t}$,
iii) $\frac{I^{\prime \prime}(t)}{I^{\prime}(t)} \sim \frac{\sigma+1}{t}$. 
Next put

$$
F(x)=\int_{a}^{x} \frac{1}{s} \int_{a}^{s} \frac{\phi(u)}{u^{2}} d u d s
$$

Then, again for $x \rightarrow \infty$

$$
\begin{array}{ll}
\text { i) } F(x) \sim \frac{1}{(\gamma-1)^{2}} \frac{\phi(x)}{x} & \text { ii) } \dot{F}(x) \sim \frac{1}{\gamma-1} \frac{\phi(x)}{x^{2}} \\
\text { iii) } \ddot{F}(x)=\left[\frac{\gamma-2}{\gamma-1}+o(1)\right] \frac{\phi(x)}{x^{3}}
\end{array}
$$

and so for $x \rightarrow \infty$,

$$
\text { i) } \frac{\phi(x) \dot{F}(x)}{F^{2}(x)} \sim(\gamma-1)^{3}, \quad \text { ii) } \frac{F(x) \ddot{F}(x)}{\dot{F}(x)^{2}}=\frac{\gamma-2}{\gamma-1}+o(1) .
$$

Consider the function

$$
Z(t)=I(t) F(x(t))
$$

From (4.11), (4.6)i) and (4.9)i) there follows for $t \rightarrow \infty$,

$$
Z(t) \sim \frac{t^{2} g(t)}{(\sigma+2)^{2}(\gamma-1)^{2}} \frac{\phi(x(t))}{x(t)} .
$$

Note that (3.4) holds for any $r<\gamma<s$ and (3.3) holds for any $s>\gamma$. Also (4.3) implies (3.5) and one can apply Theorem 3.1 and Proposition 1.1 to the both sides integrals in (3.7). This yields

$$
\frac{\phi(x(t))}{x(t)} \asymp\left(t^{2} q(t)\right)^{-1} .
$$

This, together with (4.12), yields

$$
0<m \leq Z(t) \leq M, \quad \text { for all } t \geq T .
$$

If $Z(t)$ is eventually monotone, being bounded, it tends to a positive constant.

Otherwise, we proceed as follows. From (4.11) one has

$$
Z^{\prime}(t)=I^{\prime} F+I \dot{F} x^{\prime}
$$

Another differentiation, using (A) and (4.14) (to eliminate $x^{\prime}$ ) gives

$$
Z^{\prime \prime}(t)=I^{\prime \prime} F+\frac{2 Z^{\prime} I^{\prime}}{I}-\frac{2\left(I^{\prime}\right)^{2}}{I} F+I \ddot{F}\left(\frac{Z^{\prime}-I^{\prime} F}{I \dot{F}}\right)^{2} I \dot{F} g \phi .
$$


Dividing by $I^{\prime} F=I^{\prime} Z / I$ and using (4.14) one obtains

$$
\begin{aligned}
\frac{Z^{\prime \prime}}{Z} \frac{I}{\left(-I^{\prime}\right)}= & \left(\frac{Z^{\prime}}{Z}\right)^{2} \frac{I}{\left(-I^{\prime}\right)} \frac{F \ddot{F}}{(\dot{F})^{2}}+2 \frac{Z^{\prime}}{Z}\left(\frac{F \ddot{F}}{(\dot{F})^{2}}-1\right) \\
& +Z\left[\frac{q}{\left(-I^{\prime}\right)} \frac{\phi \dot{F}}{F^{2}}\right]+\frac{I^{\prime \prime}}{\left(-I^{\prime}\right)}+2 \frac{I^{\prime}}{I}-\frac{I^{\prime}}{I} \frac{F \ddot{F}}{(\dot{F})^{2}},
\end{aligned}
$$

and finally using (4.7) and (4.10), the following asymptotic equality is obtained

$$
\begin{aligned}
\frac{Z^{\prime \prime}}{Z} \frac{I}{\left(-I^{\prime}\right)}= & \left(\frac{Z^{\prime}}{Z}\right)^{2} \frac{I}{\left(-I^{\prime}\right)} \frac{F \ddot{F}}{(\dot{F})^{2}}+2 \frac{Z^{\prime}}{Z}\left(\frac{F \ddot{F}}{(\dot{F})^{2}}-1\right) \\
& +\frac{1}{t}\left[-Z(\gamma-1)^{3}(\sigma+2)+\left(\frac{\sigma+\gamma+1}{\gamma-1}+o(1)\right)\right] .
\end{aligned}
$$

Now, let $\left\{t_{n}\right\}$ and $\left\{t_{n}^{\prime}\right\}$ be, respectively, the sequences of maxima and of minima of $Z(t)$. Note that $Z^{\prime}\left(t_{n}\right)=0, Z^{\prime \prime}\left(t_{n}\right) \leq 0, Z^{\prime}\left(t_{n}^{\prime}\right)=0$ and $Z^{\prime \prime}\left(t_{n}^{\prime}\right) \geq 0$ for $n=1,2, \ldots$ Taking the limit as $n \rightarrow \infty$ in (4.15) along the sequence $\left\{t_{n}\right\}$, one easily find that

$$
\limsup _{n \rightarrow \infty} Z\left(t_{n}\right) \leq \frac{\sigma+\gamma+1}{(\gamma-1)^{4}(\sigma+2)} \Rightarrow \limsup _{t \rightarrow \infty} Z(t) \leq \frac{\sigma+\gamma+1}{(\gamma-1)^{4}(\sigma+2)} .
$$

Likewise, taking the limit along the sequence $\left\{t_{n}^{\prime}\right\}$, one obtains

$$
\liminf _{n \rightarrow \infty} Z\left(t_{n}^{\prime}\right) \geq \frac{\sigma+\gamma+1}{(\gamma-1)^{4}(\sigma+2)} \Rightarrow \liminf _{t \rightarrow \infty} Z(t) \geq \frac{\sigma+\gamma+1}{(\gamma-1)^{4}(\sigma+2)} .
$$

Thus, even in the case where $Z^{\prime}(t)$ changes sign infinitely for large $t$ it follows that $Z(t)$ converges to a finite limit as $t \rightarrow \infty$ :

$$
\lim _{t \rightarrow \infty} Z(t)=\frac{\sigma+\gamma+1}{(\gamma-1)^{4}(\sigma+2)}>0 .
$$

The positivity follows from (4.3).

This fact, combined with (4.12), shows that

$$
\frac{\phi(x(t))}{x(t)} \sim c\left(t^{2} q(t)\right)^{-1}, \quad t \rightarrow \infty,
$$

for some constant $c>0$, which, in view of Propositions 1.3 and (1.6), means that $x(t)$ is a regularly varying function of index $\rho=(\sigma+2) /(1-\gamma)$. The constant $c$ is computed in a).

This completes the proof of $b$ ). 
It is of importance to emphasize that Theorem 4.1 is not a full generalization of Theorem 2.2 in [19] where $\phi(x)=x^{\gamma}, \gamma>1$, since in (4.3) we assume $s>\gamma$. However, if $L(x)$ is almost decreasing, then it is obvious that the condition (3.3) holds for $s=\gamma$, so one may take in (4.3) $s=\gamma$, which makes it also necessary.

Thus we have the following

Corollary 4.1. If in (4.1) in addition to (4.2) we suppose that $L(x)$ is almost decreasing, then all existing (positive) increasing solutions $x(t)$ of equation (A) of the class (1.4) (ii) are regularly varying of index $\rho>1$ with $\rho$ given by (4.4) if and only if (4.2) is satisfied and all such solutions have the same asymptotic behavior for $t \rightarrow \infty$ given by (4.5).

The remaining case $x(t) \in \mathrm{RV}(1)$ is resolved by the following:

Theorem 4.2. a) If we assume in (4.1) for $L(x(t))$ that for $t \rightarrow \infty$, $L(t \xi(t)) \sim L(t)$ with $\xi(t) \in \mathrm{SV}$ then the condition

$$
\text { (i) } \sigma=-\gamma-1
$$$$
\text { (ii) } \int_{a}^{\infty} g(u) \phi(u) d u=\int_{a}^{\infty} u^{-1} \ell(u) L(u) d u<\infty
$$

is a necessary one for all positive increasing solutions $x(t)$ of (4.1) such that $x(t) / t \rightarrow \infty, t \rightarrow \infty$ to be nontrivial regularly varying of index 1 . All these solutions have the same asymptotic behavior for $t \rightarrow \infty$

$$
x(t) \sim t\left((\gamma-1) \int_{a}^{\infty} g(s) \phi(s) d s\right)^{-1 /(\gamma-1)} .
$$

b) If we assume in (4.1) for $L(x)$ to be almost increasing the condition (4.16) is also a sufficient one.

Proof. a) In this case in view of $x(t)=t \xi(t)$ and the hypotheses on $L(x(t))$, we may repeat the argument used in the proof of Theorem 4.1a), to obtain the statement and formula (4.17) by application of Theorem 2.2. II (the only if part) in [19].

b) Assume (4.16). Bearing in mind assumption on $L(x)$, we may take in the right-hand side of (3.8), $r=\gamma$ to obtain

$$
\frac{\phi(t y(t))}{y(t)} \leq M t^{\gamma}\left\{\int_{t}^{\infty} u^{-1} l(u) d u\right\}^{-1} .
$$

Integrate equation (3.2) over $(T, t)$, use estimate (4.18) to obtain, since $y(t)$ is increasing to $\infty$,

$$
0<\frac{t y^{\prime}(t)}{y(t)} \leq \frac{T^{2} y^{\prime}(T)}{t y(t)}+\frac{M}{t} \int_{T}^{t} l(u)\left(\int_{u}^{\infty} z^{-1} l(z) d z\right)^{-1} d u .
$$


But, due to Proposition 1.1(ii) and (iii) the right-hand side of the preceding inequality is $o(1)$ so that $t y^{\prime}(t) / y(t) \rightarrow 0$, as $t \rightarrow \infty$, whence due to Proposition 1.4, $y(t) \in \mathrm{SV}$. It is also nontrivial since it tends to infinity as $t \rightarrow \infty$.

Remark 4.1. The restriction in Theorem 4.2 a) $L(t \xi(t)) \sim L(t)$ is true for e.g.

$$
L(t)=\prod_{k=1}^{N}\left(\log _{k} t\right)^{\alpha_{k}} \quad \alpha_{k} \in \mathbf{R}
$$

but not for

$$
L(t)=\exp \left(\prod_{k=1}^{N}\left(\log _{k} t\right)^{\beta_{k}}\right) \quad \beta_{k} \in(0,1) .
$$

Here $\log _{k+1} t=\log \log _{k} t$, and $\log _{1} t=\log t$.

Remark 4.2. Our results give also useful information about asymptotic form of the equation

$$
\left(p(t) x^{\prime}\right)^{\prime}=g(t) \phi(x)
$$

with $p(t) \in \mathrm{RV}(\lambda), g(t) \in \mathrm{RV}(\sigma), \phi \in \mathrm{RV}(\gamma), \gamma>1$ in the case $\int_{a}^{\infty} 1 / p(t) d t=$ $\infty$. In fact, making the change of new independent variable $\tau=P(t)$, where $P(t)=\int_{a}^{t} 1 / p(s) d s$, and new function $X(\tau)=x(t)$ equation $(\mathrm{B})$ is transformed into

$$
\frac{d^{2} X}{d \tau^{2}}=Q(\tau) \phi(X), \quad \text { where } Q(\tau)=p\left(P^{-1}(\tau)\right) g\left(P^{-1}(\tau)\right)
$$

Applying Theorem 4.1. and 4.2. to the equation (4.19) and returning to the original independent variable and the original function one obtain necessary and/or sufficient conditions for all increasing solutions of the equation (B) satisfying $\lim _{t \rightarrow \infty} x(t)=\lim _{t \rightarrow \infty} p(t) x^{\prime}(t)=\infty$ to be regularly varying (for more details, see [18, Section 3]). Moreover, the asymptotic behavior of any such solution could be determined.

For the case $\int_{a}^{\infty} 1 / p(t) d t<\infty$, similar transformation is not possible, so results should be obtained directly and thus, could be the subject of further research.

\section{Examples}

Examples illustrating the main results will be presented. 
Example 5.1. Consider differential equation (A) with

$$
\begin{aligned}
& \phi(x) \sim x^{\gamma} \log \left(x^{2}+1\right), \quad x \rightarrow \infty \quad \text { and } \\
& q(t) \sim \frac{3}{4} r(t) \frac{(\log t)^{(1-\gamma) / 2}}{t^{(3 \gamma+1) / 2} \log \left(t^{3} \log t+1\right)}, \quad t \rightarrow \infty,
\end{aligned}
$$

where $\gamma>1$ and $r(t)$ is a continuous function on $[e, \infty)$ such that $\lim _{t \rightarrow \infty} r(t)$ $=1$.

The function $q(t)$ is a regularly varying function of index $\sigma=-(3 \gamma+1) / 2$, which satisfies $\sigma<-s-1$, for some $s \in(\gamma,(3 \gamma-1) / 2)$. Then, there exists a solution $x(t)$ of equation (A) of the class (1.4)-(ii) by Theorem 2.2. It is easy to check that

$$
\frac{t^{2} q(t)}{\rho(\rho-1)} \sim \frac{\left(t^{3} \log t\right)^{(1-\gamma) / 2}}{\log \left(t^{3} \log t+1\right)}, \quad t \rightarrow \infty .
$$

Therefore, from Theorem 4.1. it follows that all increasing solutions $x(t)$ of equation (A) such that $x(t) / t \rightarrow \infty$ as $t \rightarrow \infty$ are regularly varying of index

$$
\rho=-\frac{\sigma+2}{\gamma-1}=\frac{3}{2}
$$

satisfying

$$
\frac{x(t)}{\phi(x(t))}=\frac{x(t)^{1-\gamma}}{\log \left(x(t)^{2}+1\right)} \sim \frac{\left(t^{3} \log t\right)^{(1-\gamma) / 2}}{\log \left(t^{3} \log t+1\right)}, \quad t \rightarrow \infty,
$$

implying that $x(t) \sim\left(t^{3} \log t\right)^{1 / 2}, t \rightarrow \infty$.

Observe that if in (5.1) instead of " $\sim$ " one has "=" and $r(t)=$ $1+4 /(3 \log t)-1 /\left(3(\log t)^{2}\right)$, then, $x(t)=t^{3 / 2} \sqrt{\log t} \in \mathrm{RV}(3 / 2)$ is an exact solution.

Example 5.2. Consider equation (A) with

$$
\begin{aligned}
& \phi(x) \sim x^{\gamma} \log \left(x^{2 / 3}+x\right) \quad \text { and } \\
& q(t) \sim \frac{3 r(t) t^{-\gamma-1}}{(\log t)^{3 \gamma-2} \log \left(t^{2 / 3}(\log t)^{2}+t(\log t)^{3}\right)}, \quad t \rightarrow \infty,
\end{aligned}
$$

where $\gamma>1$ and $r(t)$ is a continuous function on $[e, \infty)$ such that $\lim _{t \rightarrow \infty} r(t)=$ 1. Clearly, the slowly varying part $L(x)=\log \left(x^{2 / 3}+x\right)$ of $\phi(x)$ fulfills condition $L(x \xi(x)) \sim L(x), x \rightarrow \infty$ for $\xi \in \mathrm{SV} \cap C^{1}\left(\boldsymbol{R}^{+}\right)$, while $q(t)$ is a regularly varying function of index $\sigma=-\gamma-1$ and

$$
\int_{t}^{\infty} q(s) \phi(s) d s \sim 3 \int_{t}^{\infty} \frac{d s}{s(\log s)^{3 \gamma-2}}=\frac{1}{1-\gamma}(\log t)^{3(1-\gamma)} \rightarrow 0, \quad t \rightarrow \infty,
$$


so that (4.16)-(ii) holds. Thus, by Theorem 4.2. all increasing solutions $x(t)$ of equation (A) such that $x(t) / t \rightarrow \infty$ as $t \rightarrow \infty$ are nontrivial regularly varying of index 1 whose asymptotic behavior is given by $x(t) \sim t(\log t)^{3}, t \rightarrow \infty$.

If in (5.2) instead of " $\sim$ " one has "=" and in particular $r(t)=$ $1+2(\log t)^{-1}$, then (A) possesses an exact nontrivial $\mathrm{RV}(1)$-solution $x(t)=$ $t(\log t)^{3}$.

Acknowledgement. Second author is supported by the Research project OI-174007 of the Ministry of Education and Science of Republic of Serbia.

\section{References}

[1] Avakumović, V. G., Sur l'équation différentielle de Thomas Fermi, Publ. Inst. Math. (Beograd), 1 (1947), 101-113.

[2] Bingham, N. H., Goldie, C. M. and Teugels, J. L., Regular Variation, Encyclopedia of Mathematics and its Applications, 27, Cambridge University Press, 1987.

[3] Djurčić, D., $\mathcal{O}$-regularly varying functions and some asymptotic relations, Publications de L'institut mathematique Nouvelle serie, tome, 61, 75 (1997), 44-52.

[4] Evtukhov, V. M., Asymptotics of solutions of nonautonomous second-order ordinary differential equations asymptotically close to linear equations, Ukrainian Math. J., 46 (2013), 1531-1552.

[5] Evtukhov, V. M. and Samoilenko, A. M., Asymptotic representations of solutions of nonautonomous ordinary differential equations with regularly varying nonlinearities, Differ. Equ., 47 (2011), 627-649.

[6] Karamata, J., Sur un mode de croissance réguliere des functions, Mathematica (Cluj), 4 (1930), 38-53.

[7] Jaroš, J. and Kusano, T., Slowly varying solutions of a class of first order systems of nonlinear differential equations, Acta Math. Univ. Comenianae, LXXXII, (2013), 265-284.

[ 8 ] Jaroš, J. and Kusano, T., Existence and precise asymptotic behavior of strongly monotone solutions of systems of nonlinear differential equations, Differ. Equ. Appl., 5 (2013), 185-204.

[9] Jaroš, J. and Kusano, T., Asymptotic behavior of positive solutions of a class of systems of second order nonlinear differential equations, Electronic Journal of Qualitative Theory of Differential Equations, 23 (2013), 1-23.

[10] Jaroš, J. and Kusano, T., On strongly monotone solutions of a class of cyclic systems of nonlinear differential equations, J. Math. Anal. Appl., 417 (2014), 996-1017.

[11] Jaroš, J. and Kusano, T., Strongly increasing solutions of cyclic systems of second order differential equations with power-type nonlinearities, Opuscula Math., 35 (2015), 47-69.

[12] Jaroš, J., Kusano, T. and Manojlović, J., Asymptotic analysis of positive solutions of generalized Emden-Fowler differential equations in the framework of regular variation, Central European Journal of Mathematics, 11 (2013), 2215-2233.

[13] Kusano, T. and Manojlović, J., Positive solutions of fourth order Emden-Fowler type differential equations in the framework of regular variation, Applied Mathematics and Computation, 218 (2012), 6684-6701.

[14] Kusano, T. and Manojlović, J., Positive solutions of fourth order Thomas-Fermi type differential equations in the framework of regular variation, Acta Applicandae Mathematicae, 121 (2012), 81-103. 
[15] Kusano, T. and Manojlović, J., Asymptotic behavior of positive solutions of odd order Emden-Fowler type differential equations in the framework of regular variation, Electronic Journal of Qualitative Theory of Differential Equations, 45 (2012), 1-23.

[16] Kusano, T. and Manojlović, J., Complete asymptotic analysis of positive solutions of oddorder nonlinear differential equation, Lith. Math. J., 53 (2013), 40-62.

[17] Kusano, T. and Manojlović, J., Precise asymptotic behavior of intermediate solutions of even order nonlinear differential equation in the framework of regular variation, Moscow Mathematical Journal, 13 (2013), 649-666.

[18] Kusano, T., Manojlović, J. and Marić, V., Increasing solutions of Thomas-Fermi type differential equations - the sublinear case, Bull. T. de Acad. Serbe Sci. Arts, Classe Sci. Mat. Nat., Sci. Math., CXLIII (2011), 21-36.

[19] Kusano, T., Manojlović, J. and Marić, V. Increasing solutions of Thomas-Fermi type differential equations - the superlinear case, Nonlinear Analysis, 108 (2014), 114-127.

[20] Kusano, T., Manojlović, J. V. and Milošević, J., Intermediate solutions of second order quasilinear ordinary differential equations in the framework of regular variation, Applied Mathematics and Computation, 219 (2013), 8178-8191.

[21] Kusano, T. and Marić, V., Slowly varying solutions of functional differential equations with retarded and advanced arguments, Georgian Math. J., 14 (2007), 301-314.

[22] Manojlović, J. and Marić, V., An asymptotic analysis of positive solutions of Thomas-Fermi type sublilnear differential equations, Mem. Differential Equations Math. Phys., 57 (2012), 75-94.

[23] Marić, V., Regular Variation and Differential Equations, Lecture Notes in Mathematics, Springer, Berlin-Heidelberg-New York, 2000.

[24] Marić, V. and Tomić, M., Asymptotic properties of solutions of the equation $y^{\prime \prime}=f(x) \phi(y)$, Math. Z., 149 (1976), 261-266.

[25] Matucci, S. and Řehák, P., Regularly varying solutions of second order difference equations with arbitrary sign coefficient, Advances in Difference Equations, Article ID 673761, 16 pages, 2010.

[26] Matucci, S. and Řehák, P., Regularly varying sequences and second order difference equations, Journal of Difference Equations and Applications, 14 (2008), 17-30.

[27] Matucci, S. and Řehák, P., Asymptotics of decreasing solutions of coupled $p$-Laplacian systems in the framework of regular variation, Annali di Matematica Pura ed Applicata, 193 (2014), 837-858.

[28] Mizukami, M., Naito, M. and Usami, H., Asymptotic behavior of solutions of a class of second order quasilinear ordinary differential equations, Hiroshima Math. J., 32 (2002), 51-78.

[29] Řehák, P., Asymptotic behavior of increasing solutions to a system of $n$ nonlinear differential equations, Nonlinear Analysis, 77 (2013), 45-58.

[30] Rehák, P., Regular variation on time scales and dynamic equations, Aust. J. Math. Anal. Appl., 5 (2008), 1-10.

[31] Řehák, P., On decreasing solutions of second order nearly linear differential equations, Boundary Value Problems, 62 (2014), 1-13.

[32] Řehák, P. and Vitovec, J., Regular variation on measure chains, Nonlinear Analysis, 72 (2010), 439-448.

[33] Seneta, E., Regularly Varying Functions, Lecture Notes in Mathematics, 508, Springer, Berlin-Heidelberg-New York, 1976.

[34] Zhang, Z. and $\mathrm{Yu}, \mathrm{J}$., The exact asymptotic behaviour of the unique solution to a singular nonlinear Dirichlet problem, J. Math. Anal. Appl., 329 (2007), 1330-1342. 
[35] Zhang, Z., The second expansion of the solution for a singular elliptic boundary value problem, J. Math. Anal. Appl., 381 (2011), 922-934.

[36] Wong, P. K., Existence and asymptotic behavior of proper solutions of a class of secondorder non-linear differential equations, Pacific J. Math., 13 (1963), 732-760.

\author{
nuna adreso: \\ Takaŝi Kusano \\ Department of Mathematics, Faculty of Science \\ Hiroshima University \\ Higashi-Hiroshima 739-8526 \\ Japan \\ E-mail: kusanot@zj8.so-net.ne.jp \\ Jelena V. Manojlović \\ Faculty of Science and Mathematics \\ Department of Mathematics \\ University of Niš, \\ Višegradska 33, 18000 Niš \\ Serbia \\ E-mail: jelenam@pmf.ni.ac.rs \\ Vojislav Marić \\ Serbian Academy of Science and Arts \\ Kneza Mihaila 35, 11000 Beograd \\ Serbia \\ E-mail: vojam@uns.ac.rs
}

(Recevita la 21-an de septembro, 2015)

(Revizita la 17-an de februaro, 2016) 